\title{
OSNR-aware Control of Optical White Boxes on Elastic Optical Networks
}

\author{
Camila A. S. Diniz ${ }^{(1, *)}$, Miquel Garrich A. ${ }^{(2,3)}$, Georgios Zervas ${ }^{(4)}$, Darli A. A. Mello ${ }^{(1)}$ \\ ${ }^{(1)}$ FEEC, University of Campinas (UNICAMP), Campinas-SP, Brazil $\quad{ }^{(2)}$ Dip. di Elettronica e Telecomunicazioni, Politecnico di Torino, Italy \\ ${ }^{(3)}$ CPqD, Campinas-SP, Brazil ${ }^{(4)}$ High Performance Networks group (HPNg), University of Bristol, Bristol, UK \\ ${ }^{(*)}$ cdiniz@decom.fee.unicamp.br
}

\begin{abstract}
Results of optical white boxes on Elastic Optical Networks with adaptive modulation format and symbol rate simulations demonstrate that synthesized nodes improve capacity under low loads while preserving performance of existing ROADMs for higher loads.

OCIS codes: (060.4265) Networks, wavelength routing; (060.4510) Optical communications.
\end{abstract}

\section{Introduction}

Elastic Optical Networking (EON) is able to extend the lifetime of already deployed optical fibers to accommodate the ever-increasing network traffic demand [1]. EON combines bandwidth-variable transponders (BVTs) and wavelength selective switch (WSS)-based node architectures capable to route channels of heterogeneous bandwidths. BVTs adjust their modulation format, symbol and code rates according to the channel and network condition [2]. For a given bandwidth, this flexibility enables an increase of the channel transmission rate. Alternatively, for a given required bit rate, BVTs can reduce the channel bandwidth and save spectral resources.

EONs require advanced optical node architectures. Recent proposals on Architecture on Demand (AoD)-based function programmable optical networks offer scalability [3] reliability [4], CAPEX [5] and power consumption (OPEX) [6] savings compared with traditional optical node architectures. However, to the best of the authors' knowledge, no studies jointly exploit the AoD benefits in EON scenarios to increase network capacity or improve spectral efficiency. In this work, we propose a BVT adjustment scheme in which OSNR gains provided by AoD raise the modulation format order leading to higher network capacity or improved spectral efficiency.

\section{AoD-based programmable optical networks}

Deployed optical networks are commonly based on broadcast-and-select (B\&S) and/or route-and-select (R\&S) reconfigurable optical add/drop multiplexers (ROADMs). Fig. 1(a) shows a B\&S (R\&S) ROADM structure composed of a splitter (WSS) stage connected in a full mesh with a WSS stage. ROADMs are characterized by hardwired fiber connections, restricted upgradeability and limited flexibility. On the contrary, AoD-based function programmable optical networks rely on nodes capable to synthesize architectures according to the traffic needs and network requirements. Fig. 1(b) shows an AoD node where modules are plugged to an optical cross-connect (OXC) backplane allowing arbitrary optical functions placement.

Prior work in the area follows the steps 1 to 7 of the flowchart shown in Fig. 1(c). First, (1) traffic matrices and/or dynamic connection requests are randomly generated for given network topologies. Then, (2) routing and (3) spectrum assignment are sequentially calculated and applied for each successive connection. After that, (4) local per-node information is used to (5) execute AoD synthesis and (6) perform node evaluation. Steps (4) to (6) can be adapted to optimize scalability [3] or power balance [7]. Finally, (7) accounts for self-healing properties [4], number of WSSs employed [5] and total network power consumption [6].

In this paper, we propose a BVT adjustment scheme, executed between steps (6) and (7), to evaluate the OSNR and optimize the modulation format (and increase the bit rate) at a given symbol rate, or optimize the symbol rate (and, thus, the required bandwidth) maintaining the transmission rate.

\section{Transmission model}

We consider Nyquist-shaped transmission within an available spectrum of $4 \mathrm{THz}$, divided into $6.25-\mathrm{GHz}$ slots. Our BVT adjustment scheme chooses the modulation format considering a required SNR to ensure operation below a pre forward error correction (FEC) BER threshold of 4x10-3. The investigated modulation formats and respective SNR thresholds (in linear scale) are presented in Tab. 1.

The signal degradation is computed at each amplification stage. Line amplifiers compensate for fiber losses after each span of length L. Node (input and output) amplifiers are placed in order to guarantee that the node input power is at least $-20 \mathrm{dBm}$ and that the output spectrum is equalized with $0 \mathrm{dBm}$ for each channel [7]. The ASE power added by each amplifier is calculated as [8]: $\quad P_{A S E}=2(G-1) h v n_{s p} B_{n}$, where $\mathrm{G}$ is the amplifier gain, $\mathrm{h}$ is the Planck's constant, $v$ is the channel carrier frequency, $n_{\text {sp }}$ is the spontaneous emission factor and $B_{n}$ is the reference bandwidth. 
Finally, we assume that digital signal processing algorithms fully compensated for linear effects. The influence of nonlinear impairments is represented by the hard limit to the launch power $(0 \mathrm{dBm})$. We also do not consider OEO regeneration, and connections with insufficient OSNR are blocked.

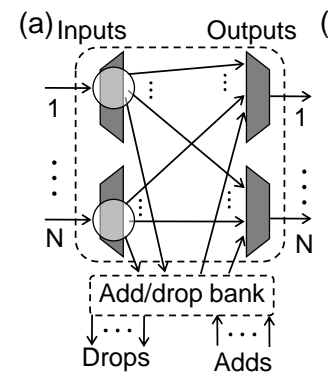

(b)

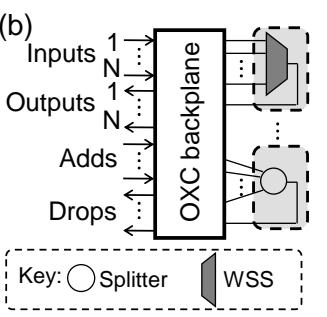

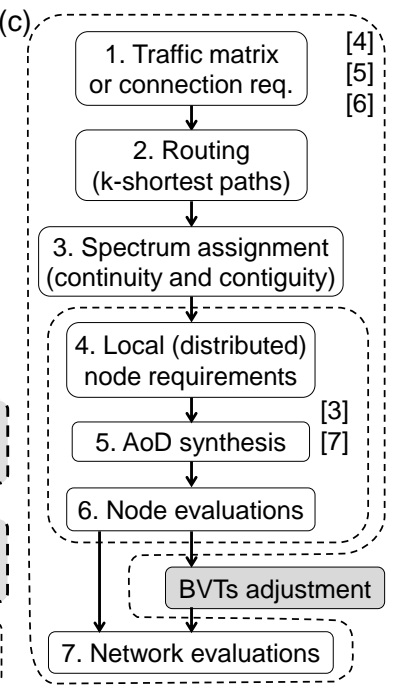

Fig. 1. (a) B\&S (splitters) R\&S (WSSs) ROADM. (b) AoD. (c) Workflow in AoD-related prior work (steps 1-7) including BVT adjustment step proposed here.
Tab. 1. Modulation formats and linear SNR threshold

\begin{tabular}{|c|c|}
\hline Mod. Format & Required SNR @ BER=4e-3 \\
\hline PM-BPSK & 3.52 \\
\hline PM-QPSK & 7.03 \\
\hline PM-8PSK & 17.59 \\
\hline PM-16QAM & 32.60 \\
\hline PM-32QAM & 64.91 \\
\hline PM-64QAM & 127.51 \\
\hline
\end{tabular}

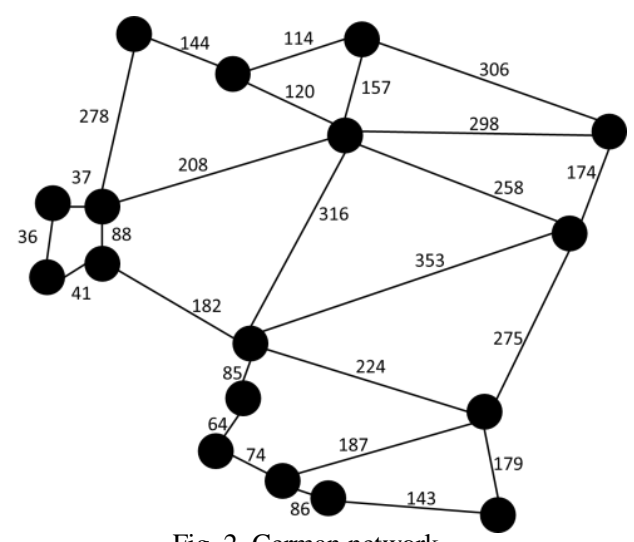

Fig. 2. German network.

\section{Simulation setup}

The AoD synthesis is computed for each network node according to an input traffic matrix [3]. The insertion loss at each AoD node is calculated based on the contributions of all components used by the connection [6]. We assume the following insertion losses: at the $\mathrm{OXC} \mathrm{L}_{\mathrm{OXC}}=2 \mathrm{~dB}$, at the WSS $\mathrm{L}_{\mathrm{wss}}=7 \mathrm{~dB}$ and at splitters/couplers $\mathrm{L}_{\mathrm{S} / \mathrm{C}}=10 \log _{10}(d g) \mathrm{dB}$, where $\mathrm{dg}$ is the node degree.

We chose the German network [9] with a total of 17 nodes and 26 links. Fig. 2 shows the network topology. Link lengths are presented in kilometers. The network has an average of 4 degrees per node and maximum of 6 . The number of degrees strongly influences the efficiency of $B \& S$ over $R \& S$, because above 5 degrees $L_{S / C}>L_{W S S}$ and R\&S outperforms B\&S.

For network performance analysis we define two different transmission scenarios: A) fixed symbol rate (and channel bandwidth) and variable bit rate and B) variable symbol rate and fixed bit rate. For both scenarios the modulation format is chosen based on the calculated link OSNR. For scenario A the symbol rate was fixed at 32 GBd. In scenario B the symbol rate is adjusted to the modulation format to provide $100 \mathrm{~Gb} / \mathrm{s}$ signal transmission with $12 \%$ FEC overhead, thus optimizing the bandwidth occupied by the channel.

\section{Simulation results}

The possibility of avoiding the insertion loss due to splitters/couplers and/or WSS's, reduces the the signal degradation caused by ASE power, increasing final OSNR for the AoD scenario. Fig. 3 shows the average OSNR gain of AoD and B\&S relative to R\&S OSNR. As the number of connections grows, the synthesis of the AoD node approaches the B\&S, narrowing the gain between both architectures. To ensure the OSNR improvement, the presented results are an average of multiple random generated traffic matrices.

The OSNR gain provided by AoD enabled higher-order modulation formats to be employed more frequently, when compared to B\&S and R\&S. Fig. 4 depicts the relative frequency of the investigated modulation formats for each node architecture with 17 connections, in scenario A. For the AoD case, 16\% of the transmitted channels employed PM-64QAM and 11\% employed PM-32QAM. B\&S, in turn, provides 6\% of connections with PM64QAM and 4\% with PM-32QAM, whereas the 90\% left uses less efficient modulation formats. R\&S uses lowerorder modulation formats because of the high input WSS loss when compared to splitter loss for low nodal degrees. 
To evaluate the network throughput of scenarios A and B, we first discarded, for the three analysed architectures, connections with insufficient OSNR. Around $6 \%$ and $20 \%$ of connections where discarded for scenario A and B, respectively. We then calculated the average bit rate per channel for B\&S, R\&S and AoD in scenario A. As shown in Fig. 5, the average bit rate increases $28 \mathrm{~Gb} / \mathrm{s}$ for 20 connections, when comparing AoD with B\&S. Moreover, the advantage of using splitters at node input becomes clear, as the difference on the average bit rate between B\&S and $\mathrm{R} \& \mathrm{~S}$ is around $40 \mathrm{~Gb} / \mathrm{s}$ in the investigated range. Evaluation for scenario B considered the average number of occupied slots versus the network throughput. As shown in Fig. 6, AoD provides a modest saving in the number of occupied slots, typically between 1 and 3 slots. Regardless of the network throughput B\&S allocates around 30\% less slots than R\&S. Again, this is due to the reduction of the insertion loss when avoiding WSS's for few degrees nodes.

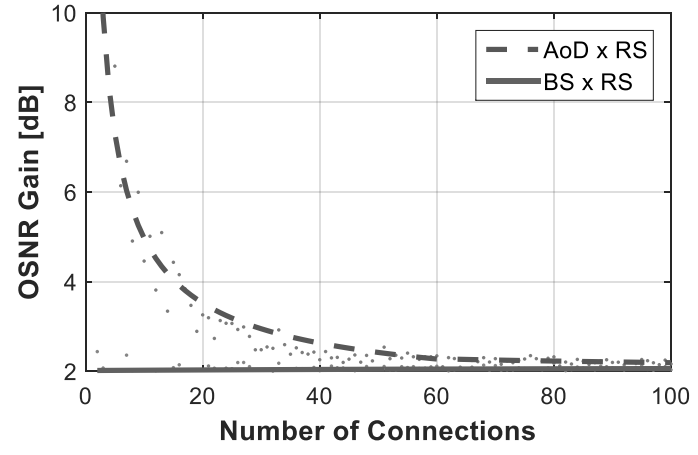

(a)

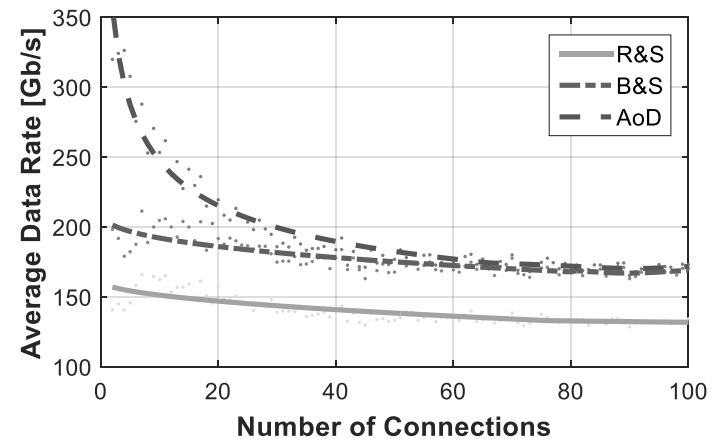

(c)

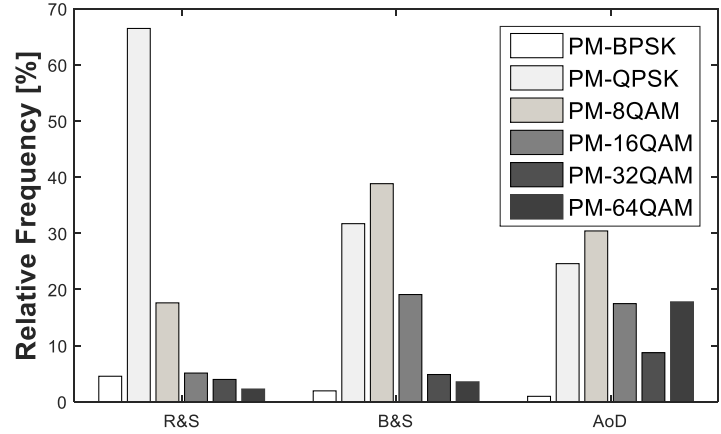

(b)

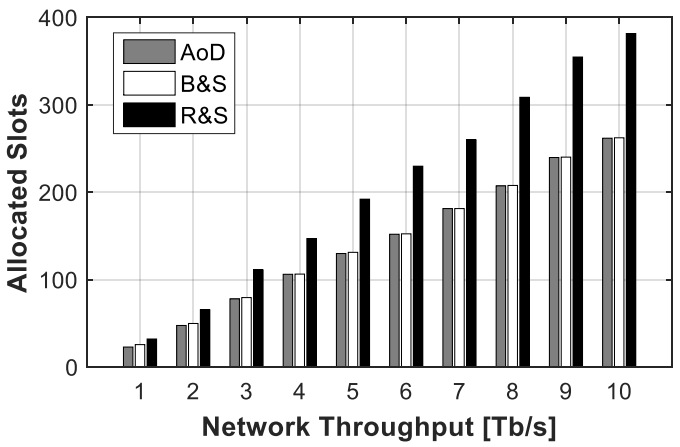

(d)

Fig. 3. (a) OSNR gain for AoD and B\&S compared to R\&S. (b) Modulation formats for 17 connections. (c) Average bit rate per connection for $32 \mathrm{GBd}$. (d) Number of occupied slots versus network throughput.

\section{Conclusions}

We investigated OSNR-aware parameter selection in AoD synthesized optical nodes. AoD has the potential to improve network throughput at low traffic loads. It also sustains same performance as per traditional ROADM architectures even on higher loads.

\section{Acknowledgements}

At Unicamp this work was supported by FAPESP and Padtec S/A. M. Garrich thanks the financial support of FUNTTEL under the project 100GETH and CNPq (grant 312047/2015-0). At Bristol the work was partially supported by EC H2020 dRedbox and EPSRC grant EP/L027070/1.

\section{References}

[1] J. Fernandez-Palacios et al, "Elastic Optical Networking: An Operators Perspective," ECOC, pp. Mo.4.2.1 (2014).

[2] D. Mello et al., "Parameter selection in optical networks with variable-code-rate transceivers," ONDM (2015).

[3] M. Garrich et al., "Architecture on Demand Design for High-Capacity Optical SDM/TDM/FDM Switching," JOCN, v.7.1, pp.21-35 (2015).

[4] M. Dzanko et al., "Experimental demonstration and benefits of self-healing hard-wired and synthetic ROADMs," OFC, pp. W1C-3 (2014).

[5] A. Muhammad et al., "Introducing flexible and synthetic optical networking: Planning and operation based on network function programmable ROADMs," JOCN, v.6.7, pp. 635-648 (2014).

[6] A. Ahmad, et al., "Switching node architectures in flexible-grid networks: A performance comparison," ONDM (2014).

[7] H. Yuan et al., "Function Placement and Configuration for Power Balanced Network Function Programmable Optical Nodes," OFC, pp. W3J-2 (2015).

[8] G. P. Agrawal, Fiber-optics communication systems, John Wiley and Sons, Inc. (2002).

[9] A. Betker, et al., "Reference transport network scenarios," MultiTeraNet Rep. (2003). 